\begin{tabular}{|l|l|} 
REVISTA Revista Educación \\
ISSN: 0379-7082 \\
ISSN: 2215-2644 \\
revedu@gmail.com \\
Universidad de Costa Rica \\
Costa Rica
\end{tabular}

\title{
Educación por habilidades: Perspectivas y retos para el sistema educativo
}

\author{
Portillo-Torres, Mauricio Cristhian \\ Educación por habilidades: Perspectivas y retos para el sistema educativo \\ Revista Educación, vol. 41, núm. 2, 2017 \\ Universidad de Costa Rica, Costa Rica \\ Disponible en: http://www.redalyc.org/articulo.oa?id=44051357008 \\ DOI: http://dx.doi.org/10.15517/revedu.v41i2.21719
}

Esta obra está bajo una Licencia Creative Commons Atribución-NoComercial-SinDerivar 3.0 Internacional. 


\title{
Educación por habilidades: Perspectivas y retos para el sistema educativo
}

Skills education: prospects and challenges for the education system

Mauricio Cristhian Portillo-Torres [1]

Ministerio de Educación Pública, Costa Rica

DOI: http://dx.doi.org/10.15517/revedu.v41i2.21719

portillozeledon@gmail.com

ORCID: http://orcid.org/0000-0001-9437-5142

\author{
Redalyc: http://www.redalyc.org/articulo.oa?id=44051357008
}

Recepción: 06 Noviembre 2015

Aprobación: 01 Diciembre 2016

\section{Resumen:}

Este ensayo analiza los proyectos DeSeCo - Programme for International Student Assessment (PISA), Assessment and Teaching of 21st Century Skills (ATC21S) y Life Skill Based Education (OMS) que han introducido la educación por habilidades en la educación general básica, tanto a nivel internacional como en Costa Rica. Ya que Ministerio de Educación Pública, a través de la Dirección de Desarrollo Curricular, impulsa una transformación curricular basada en un currículo por habilidades, se revisan los fundamentos teóricos de estos proyectos. A partir de estos se presentan los retos que supone, para el sistema educativo costarricense, una educación por habilidades. Los cinco retos señalados son: la construcción de mapas de progreso sólidos sobre las habilidades, sistemas de evaluación más formativos que sumativos, trayectorias escolares basadas en el dominio demostrado y no en la nota, la variable del tiempo en la diversidad y los apoyos a tiempo de acuerdo con las necesidades de cada estudiante. También se indican algunos temas pendientes que deben considerarse para la implementación de esta transformación curricular.

Palabras Clave: Desarrollo de las habilidades, habilidades para la vida, habilidad.

\section{Aвstract:}

This paper analyzes the Project DeSeCo - Programme for International Student Assessment (PISA), Assessment and Teaching of 21st Century Skills (ATC21S) and Life Skill Based Education (WHO). There is an ongoing process to change the current curriculum to a skill-based curriculum and an analysis of the theoretical foundations of these three projects are mentioned. Based on these assumptions there are identified five challenges for the educational system: the construction of solid progress maps for skills, more formative than summative assessment, trajectory of learning based on mastery and not in marks, time variable in diversity, and differentiated support based on individual learning needs. Also, there are some issues that must be considered in order to implement this curriculum change.

KEYWORDS: skills development, life skills, ability.

La educación tradicional busca el desarrollo de tres habilidades en el estudiantado: la escritura, la lectura y la aritmética. La buena educación se asocia con el desarrollo del saber leer, saber escribir y saber contar. Al mismo tiempo, el sistema educativo tradicional se basa en la relación unidad-tiempo que busca garantizar una mínima exposición a los contenidos por parte de estudiantes (Sturgis, 2015). El ideal del sistema tradicional es abarcar la mayor cantidad de contenidos posibles en el tiempo establecido.

La educación tradicional basada en la unidad-tiempo se refleja en los modelos curriculares lineales que han dominado el quehacer educativo durante varias décadas tanto en Costa Rica como en otros países. Estos modelos curriculares siguen diseños lineales como los de Bobbitt y Tyler, a saber, selección de objetivos, contenidos de aprendizaje, experiencias de aprendizaje y evaluación de los propósitos (Ornstein y Hunkins, 2009).

\section{NotAS DE AUTOR}

[1] Master en Educación con acentuación en procesos de enseñanza aprendizaje del Instituto Tecnológico y de Estudios Superiores de Monterrey (ITESM). Licenciado en Docencia de la Teología de la Universidad Estatal a Distancia (UNED). Posgrado en Juventud, Educación y Trabajo (FLACSO, Argentina). Actualmente trabaja en investigación en la Dirección de Desarrollo Curricular del Ministerio de Educación Pública (MEP). Ha sido docente en colegios privados y públicos de primaria y secundaria. Su principal tema de investigación ha sido la permanencia y prevención del abandono escolar o exclusión educativa, labor por la cual ganó concurso sobre deserción Graduate xxi del Banco Interamericano de Desarrollo (BID). 
Las tendencias actuales en la cultura, en la sociedad, en la economía, en la tecnología y en la política demandan cambios en los sistemas educativos. Existe una agenda de reforma que recorre todo el planeta y que Sahlberg (2006) sintetiza de esta manera: altos estándares de aprendizaje, currículo centralizado, priorización de lecto-escritura, indicadores y logros de objetivos, una evaluación alineada a los estándares e indicadores y la contabilidad del rendimiento. En armonía con esta agenda, los nuevos modelos curriculares se diseñan de acuerdo con los estándares educativos o en resultados de aprendizaje. Estos estándares o resultados del currículo se expresan a través de competencias o habilidades consideradas esenciales para la ciudadanía del siglo XXI.

La búsqueda de las competencias o habilidades esenciales para el siglo XXI ha generado una rica reflexión mundial donde no siempre son claros los límites entre lo que es una habilidad o una competencia. Una revisión internacional nos permite encontrar términos como "competencias básicas", "habilidades para la vida", "habilidades del siglo 21" o "competencias del siglo 21" (MECD, 2013).

El propósito de este documento es analizar los fundamentos teóricos de tres perspectivas sobre el tema de habilidades aplicadas en la educación básica, a fin de comprender los cambios y retos que supone para la educación tradicional. Cada uno de estos enfoques ha sido referente a nivel nacional. En primer lugar se desarrollan las competencias básicas de acuerdo con el Proyecto DeSeCo de la Organización para la Cooperación y el Desarrollo Económicos (OCDE), y que ha tenido una amplia difusión gracias al Programa Internacional para la Evaluación de Estudiantes (PISA). En segundo lugar, se describe el proyecto Assessment \& Teaching of 21st Century Skills (ATC21s) sobre las habilidades del siglo XXI. En tercer lugar, se hace referencia a Life Skill Based Education (Educación basada en habilidades para la vida) desarrollado por las agencias de las Naciones Unidas en sus diversos proyectos educativos a nivel mundial.

Estas experiencias y sus sustentos teóricos son relevantes para entender la transformación curricular que actualmente realiza la Dirección de Desarrollo Curricular del Ministerio de Educación Pública con el lema "Educar para una Nueva Ciudadanía”, ya que la transformación curricular propone un diseño del currículo a partir de trece habilidades: pensamiento sistémico, pensamiento crítico, aprender a aprender, resolución de problemas, creatividad e innovación, ciudadanía global y local, responsabilidad personal y social, estilos de vida saludables, vida y carrera, colaboración, comunicación, apropiación de tecnologías digitales y manejo de la información (MEP, 2015).

\section{DEFINICIÓN PRELIMINAR SOBRE HABILIDAD}

No existe una única definición sobre qué es una habilidad. Este término está siendo utilizado en los diferentes países sin tener un consenso. La definiciones dependen del lente desde el cual se mira: el trabajo, la comunicación, la tecnología, la sociedad, lo psicosocial, la política. Lo cierto es que la habilidad es un constructo social (Rigby y Sanchis, 2006).

En las naciones de tradición anglosajona, la habilidad o "skill" se usa de forma equivalente a "saber cómo" o técnica (Clarke y Winch, 2006). Aplicado de esta forma se entiende que una persona tiene o no cierto grado de habilidad para realizar algo. En el ámbito laboral la habilidad se entiende como una combinación de conocimientos de materiales y procesos con destrezas manuales requeridas para lleva a cabo una actividad productiva. Es decir, la habilidad representa una propiedad individual, una destreza física y mental para realizar una tarea en el proceso del trabajo (Clarke y Winch, 2006). La habilidad solo puede ser demostrada en el rendimiento (haciendo algo), mientras que el conocimiento puede obtenerse por medios más abstractos, como la conversación. Por eso, la habilidad se identifica como conocimiento práctico o técnico, la capacidad de aplicar conocimiento teórico en un contexto práctico. Esta forma de entender la habilidad se acerca al concepto de competencia.

Otra forma alternativa de ver las habilidades es la que tiene la Organización Mundial de la Salud (OMS) que las utiliza en un contexto más amplio que el trabajo. Las habilidades para la vida se definen como la 
capacidad de un comportamiento del individuo positivo y adaptable de forma efectiva a las demandas y retos de todos los días (WHO, 1997).

Patrick Griffin, líder del proyecto Assessment and Teaching of 21st Century Skills (ATC21s), define las habilidades como acciones que las personas pueden realizar. La competencia abarca la calidad y capacidad de transferencia de una acción en el tiempo y en el contexto. Es decir, nadie aplica una habilidad con la misma destreza todo el tiempo, el desempeño se ajusta de acuerdo con las exigencias del momento y del contexto. Por tanto, la competencia es la capacidad de la persona para ajustar el rendimiento de la habilidad según la demanda del contexto (Griffin y Care, 2014).

Michael W. Connell, Kimberly Sheridan y Howard Gardner (2003) proponen que las habilidades son capacidades biopsicológicas que tienen las personas y las competencias, el conocimiento y las capacidades que son valoradas por la sociedad y la cultura. Es decir, las habilidades son de naturaleza individual y las competencias de naturaleza social. También argumentan que existen dos cualitativamente distintos tipos de habilidades humanas (modular e integrativa) que corresponden a dos categorías de problemas (tareas modulares y situaciones integradas, respectivamente). En el caso de las tareas estas se enfocan en habilidades específicas, en contraste, las situaciones, que son por naturaleza complejas, requieren de una orquestación de capacidades.

En resumen, las habilidades tienen su fundamento en lo que las personas son capaces de hacer desde sus condiciones neurofisiopsicológicas; estas pueden ser específicas, cuando son requeridas en cierta tareas, e integrativas cuando se realizan en situaciones complejas.

\section{Competencias básicas DEL SIGLO XXI}

El enfoque basado en competencias tiene sus orígenes en países anglosajones (Estados Unidos, Canadá, Reino Unido, Australia y Nueva Zelanda) en la década de los sesentas. Sus raíces están en el mundo laboral, en el análisis de las tareas o conductas requeridas para los puestos de trabajo y el desarrollo profesional. En un principio se habla de diversos tipos de competencias: laborales y profesionales, blandas y duras, genéricas y específicas, básicas y docentes. Cada una en relación con diversos contextos donde son aplicadas (Lozano y Herrera, 2013).

El salto de las competencias del mundo laboral al mundo educativo se da por medio de la educación técnica, a través de la formación profesional con instrumentos como DACUM, AMOD y SCID. La Organización para la Cooperación y el Desarrollo Económico (OCDE), a través de dos importantes iniciativas, la definición y selección de competencias (DeSeCo) y el Programa Internacional para la Evaluación de Estudiantes (PISA) ha dado un impulso a una educación basada en competencias entre sus países miembros.

El marco conceptual del proyecto DeSeCo clasifica las competencias claves en tres amplias categorías (OCDE, 2005): el uso interactivo de herramientas (uso del lenguaje, los símbolos y los textos, uso interactivo del conocimiento y la información; uso interactivo de la tecnología), la interacción con grupos heterogéneos (relacionarse bien con otras personas, manejar y resolver conflictos, cooperar y trabajar en equipo,) y la acción autónoma (actuar dentro del contexto del gran panorama, formar y conducir planes de vida, defender y asegurar derechos).

En el caso de las pruebas escritas de PISA, estas miden las competencias de lectura, de comprensión matemática y pensamiento científico (OCDE, 2005). PISA busca identificar el grado de reflexión que muestra cada estudiante en relación con el conocimiento y el aprendizaje "que sirva de base para el marco de competencia" (OCDE, 2005, p.16). Es decir, en el caso de la evaluación de la lectura, se mide si la persona puede ubicar e interpretar la información, además de reflexionar sobre ella y evaluar lo que ha leído (habilidades cognitivas). El desempeño de la lectura se ubica en seis niveles de destreza, cada nivel refleja un grado de complejidad de los procesos mentales utilizados por estudiantes en el procesamiento de la información. Otros aspectos como actitudes y disposiciones se preguntan en un cuestionario aparte. 
PISA tiene un enfoque meramente cognitivo e individual en la evaluación de competencias: se centra en la evaluación de las estrategias, de los procesos mentales aplicados por estudiantes en la resolución de cada ítem. Para solventar esto, en el 2015 se introdujo en la prueba la evaluación de la resolución colaborativa de problemas, una habilidad compuesta que implica habilidades cognitivas y sociales (OCDE, 2013).

Costa Rica ha participado en dos ocasiones en las pruebas PISA (2009 y 2012). De acuerdo con los resultados del 2012, cuya medición principal fue las matemáticas, el 56,7\% de estudiantes costarricenses de 15 años se ubicó en el nivel 1 o por debajo de él. Esto significa que la mayoría de estudiantes solo fueron capaces de responder preguntas claramente definidas (Programa Estado de la Nación, 2013).

\section{HABILIDADES DEL SIGLO XXI}

En este nombre se incluyen diversas iniciativas en las que participan gobiernos, organizaciones, fundaciones, universidades y sectores económicos que buscan la definición de las habilidades del siglo XXI. Estas habilidades son fruto de investigación y consulta a diversos sectores, sobre todo en el ámbito económicolaboral. Se parte del consenso de que las economías más avanzadas demandan nuevas habilidades de parte de las personas, y que la habilidades rutinarias y repetitivas son propias de economías menos desarrolladas (Trilling y Fadel, 2009).

Algunos estudios argumentan que el término habilidad es preferible al término de competencia, pues este proviene del mundo de los negocios y de la empresa. La retórica de la competencias del siglo XXI es una visión economicista de la educación, cuya meta es preparar personal trabajador para las economías del conocimiento de acuerdo con la crítica. Se dice que el discurso de competencias exagera la relevancia de las competencias relacionadas con el ámbito laboral (OECD, 2010). Sin embargo, este argumento no es del todo válido, pues el enfoque economicista también está presente en las habilidades del siglo XXI.

Assessment and Teaching of 21st Century Skills (ATC21s) es un ejemplo de los diversos intereses representados en la definición de habilidades, incluso económicos. El proyecto que se desarrolló durante 2009-2012 fue liderado por la Universidad de Melbourne (Australia) con la participación de gobiernos (Australia, Costa Rica, Finlandia, Holanda, Singapur y Estados Unidos), organizaciones intergubernamentales (BID, IEA, OECD, ONU, UNESCO, BM), grupos de investigación, instituciones educativas y empresas comerciales (INTEL, CISCO, MICROSOFT). El objetivo del proyecto fue la investigación, el desarrollo de métodos y tecnologías para el apoyo de la enseñanza y la evaluación de aprendizaje del siglo XXI alrededor del mundo (Act21s, 2011).

La primera tarea de ACT21s fue la definición de las habilidades que eran esenciales para una adecuada respuesta a los cambios en la vida, en el aprendizaje y el trabajo, debido a la tecnología. Estas habilidades debían permitir demostrar nuevas formas de pensar, nuevas formas de trabajar, nuevas herramientas para el trabajo y la vida en un mundo cambiado por la tecnología. En concreto, se seleccionaron las siguientes habilidades: creatividad e innovación, pensamiento crítico, resolución de problemas, aprender a aprender, vida y carrera, responsabilidad personal y social, ciudadanía local y global, apropiación de las tecnologías digitales, manejo de la información, comunicación y colaboración (Griffin y Care, 2014). El proyecto desarrolló, especialmente, una forma para validar una medición para resolver, colaborativamente, problemas (collaborative problem solving), que requiere de un conjunto de habilidades de tipo social y cognitivo.

Costa Rica participó para la validación de los instrumentos para América Latina y gestionó el proyecto desde Despacho del Ministro y con colaboración de la Fundación Omar Dengo. Un resumen de la participación de Costa Rica en el proyecto puede leerse en el capítulo doce del libro Assessment and Teaching of 21st Century Skills: Methods and Approach. 


\section{HABILIDADES PARA LA VIDA}

En la Conferencia Mundial de Educación Para Todos (Jomtien 1990) se dio relevancia a la educación y, en particular, a las habilidades que son apropiadas para la vida (Unesco, 2004). Ha sido, sobre todo, la Organización Mundial de Salud (OMS) la que ha liderado la reflexión y la aplicación de las habilidades para la vida en las agencias de las Naciones Unidas. Originalmente promovidas por el Programa de Salud Mental de la OMS desde el año 1993, estas habilidades son definidas como competencias psicosociales. Actualmente también estas habilidades son un referente para otras organizaciones como UNICEF a través de su metodología Life Skill-Based Education para la prevención del SIDA (UNICEF, 2005).

Una de las primeras definiciones dadas por WHO (1997) sobre habilidades para la vida es la siguiente: "son habilidades para un comportamiento positivo y de adaptación, que permite a los individuos lidiar efectivamente con la demandas y los retos de todos los días" (p.1). Las habilidades que se definieron como básicas para la salud y el bienestar de la niñez y adolescentes fueron: toma de decisiones, resolución de problemas, pensamiento creativo, pensamiento crítico, comunicación efectiva, habilidades para las relaciones interpersonales, empatía, manejo de las emociones, manejo del estrés, auto consciencia.

Ante la necesidad de tener un marco teórico común sobre las habilidades para la vida, las agencias de Naciones Unidas (UNESCO, UNICEF, WHO, FAO y otras) se han reunido en varias ocasiones (1999, 2004) para conversar sobre el tema. Algunas de las reflexiones que se dieron en los paneles de trabajo (UNESCO, 2004) son:

- Habilidades para la vida y desarrollo humano sostenible: La educación de calidad debe impartir tanto habilidades manuales, así como habilidades psico-sociales que ayuden a la personas a pensar, sentir, actuar e interactuar como individuos y como miembros partícipes de la sociedad. Las habilidades para la vida son difíciles de medir.

- Significado de habilidades para la vida: Es un proceso cuyo objetivo es ayudar a la gente a alcanzar lo que considera importante. Existe el acuerdo de que las habilidades para la vida son una nueva cultura de aprendizaje. Estas habilidades no deben verse de forma aislada sino como una preparación para la vida, como una culminación de la combinación de habilidades manuales y psicosociales. Implica el desarrollo de conocimientos y actitudes.

- Descripción de habilidades para la vida: Balance entre conocimiento, valores, actitudes y habilidades. Usa métodos de enseñanza interactivos y centrados en el estudiantado, se basa en las necesidades de los sujetos participantes (situaciones cotidianas), y la satisfacción de sus derechos.

- Habilidades para la vida y educación basada en habilidades: La perspectiva de habilidades para la vida ha sido ampliamente utilizada en la educación para la salud. Esta visión es complementaria a una educación basada en habilidades.

- Habilidades para la vida y sus cuatro dimensiones: Debe abarcar las dimensiones individual, social, cognitiva/reflexiva e instrumental.

Para los organismos de las Naciones Unidas, las habilidades para la vida son esenciales para la prevención primaria en temas como (WHO, 1999): embarazo adolescente, SIDA, violencia, abuso infantil, suicidio, abuso de alcohol, tabaco y otras sustancias, racismo, conflictos y temas ambientales. De ahí la insistencia que estas habilidades sean enseñadas en las escuelas y en los colegios, tanto dentro del aula, como fuera de ella.

Estas habilidades han sido agrupadas en tres categorías: las cognitivas para el análisis y uso de la información, las personales para el desarrollo de la acción personal y la gestión de uno mismo o una misma, y las interpersonales para comunicarse e interactuar eficazmente con las demás personas (ver Tabla 1). 
TABLA 1

Clasificación de las habilidades para la vida

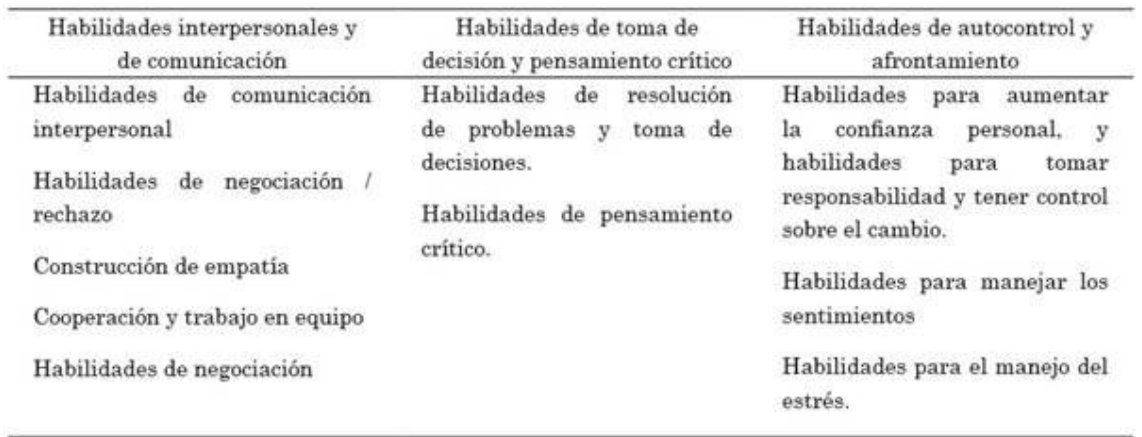

Nota: Elaboración propia a partir del Unicef (2005).

En Costa Rica, las habilidades para la vida a nivel de educación básica se han trabajado en los programas y proyectos del Departamento de Orientación del Ministerio de Educación Pública (MEP) (Carballo, 2015). La revisión de los programas de estudios vigentes para orientación permiten observar la idoneidad del desarrollo de estas habilidades de acuerdo con las tareas y responsabilidades de este servicio educativo: cuidado del cuerpo y la salud integral, desarrollo de la personalidad, bienestar individual y social, valores personales, sociales y espirituales; exploración de aptitudes, habilidades e intereses, y desarrollo de habilidades intelectuales (MEP, 2008 y 2009).

\section{Marco teórico de las habilidades DEL SIgLO XXI}

Un aspecto importante para comprender las diversas perspectivas sobre habilidades de los anteriores proyectos es conocer los fundamentos teóricos de cada uno. Algunos referentes son más psicométricos, como los utilizados por PISA, y otros más fenomenológicos como el de los hermanos Dreyfus mecionados en el proyecto ATC21s. También existen referentes teóricos comunes como es la taxonomía actualizada de Bloom para la descripción de los procesos cognitivos (Anderson, Krathwohl y Bloom, 2001).

En el caso de PISA, el desarrollo de las habilidades se establece de acuerdo con el puntaje en los ítems de las pruebas, los cuales corresponden a diferentes niveles de procesos cognitivos. Se trata de una forma estadística de establecer la adquisición de las habilidades. La relación de habilidades y puntaje se basa en los trabajos del matemático danés Georg Rasch (1901-1980), quien creó un modelo para la interpretación de ltems en una prueba psicométrica. Así mismo, en las investigaciones de Robert Glaser (1921-2012), psicólogo estadounidense, sobre la evaluación criterial para las pruebas de rendimiento educativo (Jornet y González, 2009).

El proyecto ATC21s tiene también como referentes a George Rasch y Robert Glaser y los combina con la teoría de la zona de desarrollo próxima de Vygotsky (ACTS21, 2011b). De acuerdo con Vygotsky, la zona de desarrollo próximo (ZDP, figura 1) es la distancia entre el nivel actual de solución de un problema de forma independiente de parte del estudiantado y el nivel de desarrollo potencial al resolver un problema determinado con la guía de una persona adulta o la colaboración con un par más capaz (Barker, 1996). La ZDP deja atrás concepciones teóricas que solo toman en cuenta el desarrollo cognitivo del estudiantado y considera lo que alguien puede lograr con la ayuda de otra persona, un aspecto importante a considerar cuando se busca evaluar la resolución colaborativa de un problema. 


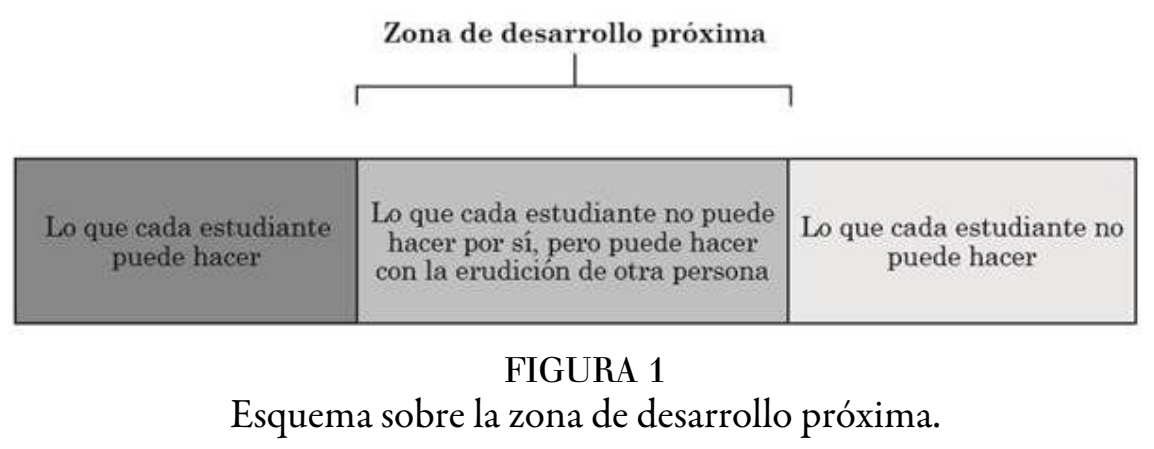

Por tanto, en el proyecto ATC21s, la evaluación busca, en primer lugar, determinar el desarrollo de la habilidad cognitiva identificando lo que cada estudiante puede realizar con el conocimiento que tiene. A partir de este punto, establecer los pasos siguientes en el desarrollo de la habilidad con la colaboración de otras personas (docente o estudiantes).

ATC21s resalta el trabajo de los hermanos Stuart Dreyfus (Universidad de Berkeley) y Hubert Dreyfus (MIT) sobre cómo se desarrolla la adquisición de una habilidad. El trabajo de ellos sirve como ejemplo del progreso empírico de las habilidades. Según estos autores, una habilidad se desarrolla en cinco estados (Dreyfus, 2004; Dreyfus y Dreyfus, 1986):

- Estado novicio: La tarea se descompone en elementos libres de contexto que el sujeto novicio puede reconocer. Al sujeto novicio se le dan reglas a seguir de acuerdo con estos elementos. Este las ejecuta sin tener un entendimiento del contexto en el que la información tiene sentido. Seguir meramente las reglas, en la vida real, produce un desempeño pobre.

- Estado principiante avanzado: El sujeto principiante comienza a ganar experiencia efectiva con situaciones reales. Así comienza a notar información adicional en relación con el contexto. Con suficientes ejemplos, el sujeto principiante aprende a reconocer estas situaciones contextuales. Todavía el aprendizaje se da en un marco independiente y analítico, siguiendo instrucciones y ejemplos.

- Estado competente: Con más experiencia, el número potencial de elementos y procedimientos que la persona puede reconocer aumenta. La persona es capaz de tomar perspectiva en lo que realiza: aquello que debe ser considerado relevante y qué puede ignorar de cada situación particular. El estudiantado aprende a limitarse a unas cuantas características y aspectos que hacen más fácil la comprensión y la toma de decisiones. La persona competente de forma natural busca reglas y procesos de razonamiento para decidir qué plan o perspectiva adoptar a fin de evitar errores. En este nivel de desempeño existe una inversión emocional en la selección de una perspectiva para la acción.

- Estado diestro: Cada vez con más nivel de involucramiento emocional en la actividad, se le vuelve difícil volver a una postura de seguir las reglas como principiante. Acepta la ansiedad de tener que elegir para avanzar en su habilidad. Las experiencias positivas o negativas emocionalmente reforzarán la perspectiva de éxito y la inhibición de las de fracaso. El seguimiento de reglas y principios es remplazado por la discriminación de las situaciones. La competencia parece desarrollarse sí y solo sí la experiencia es asimilada de esta forma no teórica.

- Estado experto: Sabe lo que hay que hacer y decide cómo hay que hacerlo. El sujeto experto no solo sabe qué hacer, debido a su amplio repertorio de discriminación de situaciones, sino también sabe inmediatamente cómo lograr el objetivo. La capacidad de hacer discriminaciones más sutiles y refinadas lo diferencia del sujeto diestro. Frente a muchas situaciones vistas como similares respecto a una misma perspectiva, el sujeto experto ha aprendido a distinguir y reaccionar de forma diferente. Aplica diferentes decisiones tácticas, analizando las situaciones y descomponiéndolas en subclases, de acuerdo con las respuestas diferenciadas de cada clase. Esto le permite una respuesta intuitiva. 
Finalmente, la iniciativa habilidades para la vida (de la OMS) solo presenta un referente para entender el ciclo de desarrollo de una habilidad (Mangrulkar, Whiatman y Posner , 2001). Este modelo se presenta a continuación (ver Figura 2).

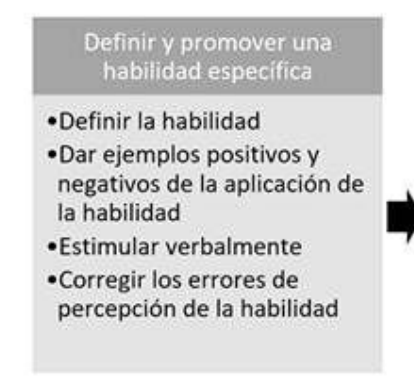

FIGURA 2

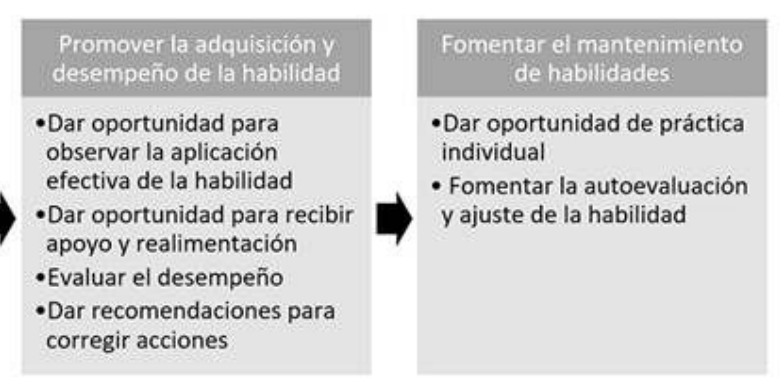

Ciclo de desarrollo de la habilidad (Mangrulkar, Whiatman y Porner, 2001)

\section{RETOS Y CRÍTICAS DE LA EDUCACIÓN POR HABILIDADES}

Como se mencionó anteriormente, la educación tradicional produce lagunas en el aprendizaje porque está basada en torno a la unidad-tiempo que garantiza una mínima exposición a contenidos, pero sin garantía de aprendizaje (Sturgis, 2015). Lo más importante para el sistema tradicional es abarcar los contenidos de aprendizaje, aunque se invisibilice cuánto aprendió realmente cada estudiante. Las cifras de reprobación y repitencia confirman que el progreso de cada estudiante no es el fin último del sistema. En cambio, la educación por habilidades tiene su principal interés en la idea del progreso de una habilidad y, por ende, en el progreso de cada estudiante en el desarrollo de la habilidad en el proceso educativo (Heritage, 2015).

El progreso del aprendizaje es la característica principal de una educación por habilidades. Heritage (2015) menciona cómo el progreso es descrito por diversos estudios como un mapa de progreso, una descripción cada vez más sofisticada de una forma de pensar sobre algo, descripciones de cómo un estudiante o una estudiante gana experticia a lo largo del tiempo. En definitiva, concebir el aprendizaje en términos de habilidad incluye la noción de continuidad y coherencia.

Un mapa de progreso de una habilidad puede diferir en el nivel de detalle de acuerdo con los niveles que se desean describir. Puede elaborarse considerando un año escolar, varios niveles o un ciclo escolar. Su objetivo debe ser proveer de suficiente información para que el personal docente pueda planificar sus clases y dar una adecuada retroalimentación a cada estudiante.

El primer reto del tránsito de la educación tradicional a una educación por habilidades es, por tanto, la construcción de mapas de progreso sólido que se sustenten en la experiencia de docentes, así como en investigaciones científicas. Los mapas de progreso permiten visualizar cómo las habilidades se desarrollan a lo largo del tiempo. Esta es una tarea que requiere de consensos.

Dada la importancia de los descriptores de los diferentes niveles de rendimiento o progreso, estos deben contar con suficiente evidencia empírica y teórica de su construcción. Jornet y González (2009) sugieren tres métodos para realizar esta tarea: métodos de juicio, métodos empíricos y métodos mixtos. Los métodos de juicio, como es el caso del juicio de personas expertas, suelen carecer de suficiente representatividad y estar limitados al contexto de quienes lo elaboran. Los métodos empíricos, por su parte, utilizan información empírica como es el caso de los resultados de test. Finalmente, los métodos mixtos combinan el juicio de personal experto y la evidencia empírica de desempeño.

En el caso de la transformación curricular "Educar para una Nueva Ciudadanía” los niveles de rendimiento de las habilidades fueron construidos de forma mixta y organizados de acuerdo con los ciclos del sistema 
educativo (MEP, 2015). Sin embargo, la mayoría de las habilidades no fueron definidas en el contexto local, ya que fueron asumidas de las propuestas por el proyecto ATC21s.

El segundo reto es la construcción de un sistema de evaluación que ponga más énfasis en la evaluación formativa y no tanto en la sumativa. Mientras que en la educación tradicional las notas pueden ocultar el verdadero progreso del estudiantado, en la educación por habilidades la evaluación formativa es la pieza clave. Esta es capaz de monitorear el progreso individual y dar retroalimentación constante a cada estudiante. Para que haya una eficaz evaluación formativa deben darse las siguientes tres condiciones: generar evidencias sobre el aprendizaje para reducir la brecha entre el desempeño actual y el deseado; dar retroalimentación al estudiantado, e involucrar al estudiantado en la evaluación y el progreso de aprendizaje (Heritage, 2015).

Una evaluación por habilidades requiere, además, de un buen conocimiento de la evaluación criterial, algo que no se menciona en el documento oficial del Ministerio de Educación Pública sobre la transformación curricular (MEP, 2015). La evaluación criterial puede tomar como referencia un estándar de desempeño como el progreso individual de cada estudiante. Definir cuál de los dos casos se aplicará en la transformación curricular es un tema pendiente.

El tercer reto es la trayectoria escolar basada en el dominio demostrado y no sobre la calificación. El sistema educativo tradicional promueve al estudiantado si este superó la nota mínima, o bien, lo reprueba si no alcanza esta nota. En cambio, en el aprendizaje por habilidades cada estudiante avanza de acuerdo con la maestría demostrada (Sturgis, 2015). Por consiguiente, la nota ya no es el único referente de logro, sino el dominio demostrado de una habilidad. A partir de esta situación, lo fundamental es dar seguimiento no al registro de notas de un estudiante, sino cómo esta persona progresa a lo largo del tiempo en el dominio de las habilidades.

Un cuarto reto, muy relacionado con el punto anterior, es la relación tiempo-aprendizaje. Como se indicó anteriormente, la relación tiempo-contenido se consideraba la unidad de medida del aprendizaje en la educación tradicional. Más contenido en menos tiempo se consideraba una señal de eficiencia y calidad. Sin embargo, la educación de habilidades cambia esta unidad de medida por tiempo-habilidad. Una habilidad se desarrolla en tiempos diferentes, lo cual no depende tanto del personal docente como del hecho de que cada estudiante aprende de forma diferente y que por diversas razones requiere de más o menos tiempo. Una frase común de esta nueva relación se expresa así: "El aprendizaje es constante, y el tiempo es la variable" (Sturgis, 2015, p. 8).

Considerar el tiempo en los procesos de aprendizajes va más allá de las actividades dentro del salón de las clases. Se requiere una revisión de los horarios, de la cantidad de contenidos que se abarcan en el currículo, la duración del año escolar y de la cantidad de estudiantes que cada docente tiene a cargo. La transformación curricular impulsada por el Ministerio de Educación Pública no hace referencia a estos factores que son muy importantes para el éxito de un currículo basado en habilidades.

Un quinto reto es cómo dar los apoyos a tiempo de acuerdo con las necesidades de cada estudiante. En la educación tradicional los vacíos de aprendizaje, las lagunas de estudiantes no suelen ser atendidos a tiempo, ya que los programas de estudio y los modelos pedagógicos se construyen con una filosofía de una medida para todos y todas. El énfasis está en presentar los mismos contenidos para todo el estudiantado, desde una concepción republicana de la igualdad que ha homogenizado el proceso de enseñanza (Dussel, 2004). Lo efectos de esta visión son evidentes en el rezago educativo. La educación por habilidades, al centrarse más en el progreso del aprendizaje por el dominio de cada estudiante, reconoce la diversidad del alumnado. Al mismo tiempo, concibe este progreso como un continuum para el estudiantado. Este continuum solo puede presentarse, si se dan, de forma oportuna, los apoyos a aquel estudiantado que presenta dificultades desde un inicio. 


\section{REFLEXIONES FINALES}

Los tres proyectos descritos, así como los fundamentos teóricos subyacentes, reafirman la idea de que la educación por habilidades es un constructo social que tiene diferentes fuentes dependientes del contexto y los intereses de los sujetos involucrados. Los orígenes teóricos pueden encontrarse en las competencias laborales, en la educación para la salud mental y física, teorías pedagógicas, uso de herramientas tecnológicas en la vida diaria y modelos psicométricos. Dependerá de los intereses del proyecto la selección de los supuestos más adecuados.

El giro hacia una educación por habilidades supone una nueva cultura de aprendizaje, cuyo fin debe ser la preparación para la vida (UNESCO 2004). No basta hablar de un currículo por habilidades sin los necesarios cambios en los valores, las prácticas educativas y la organización administrativa. Comprender que cada estudiante progresa a diferentes ritmos en el dominio de una habilidad implica saber atender oportunamente sus necesidades y la personalización de las trayectorias escolares. Este cambio en la cultura y en la práctica es, sin duda, el mayor reto del sistema educativo tradicional.

Si bien el documento oficial del Ministerio de Educación Pública orienta sobre algunos aspectos de la transformación curricular sobre habilidades, omite hacer referencia a factores importantes para que la propuesta curricular sea exitosa: el manejo del tiempo, cantidad de estudiantes por docente, el tipo de evaluación criterial, organización de los servicios de apoyo, entre otros.

La educación por habilidades cambia la visión fragmentada de la educación de acuerdo con áreas específicas y especializadas del saber o de dominios del conocimiento (conceptual, procedimental, actitudinal). La habilidad de resolución colaborativa de problemas, por ejemplo, requiere no solo de procedimientos o conceptos básicos, implica el desarrollo de habilidades psicosociales donde los grupos de estudiantes aprenden a negociar y comunicarse con otros grupos o personas. Si bien este cambio es mucho más apegado a cómo en la vida diaria los seres humanos solucionamos problemas en grupos, es todo un reto para el sistema de evaluación.

En conclusión, la agenda del cambio hacia una educación por competencias o habilidades (en este escrito se ha utilizado el concepto habilidad) conlleva un cambio significativo para los sistemas educativos tradicionales. No se trata solamente de importar y entender nuevos conceptos en educación, sino de un cambio en la filosofía, los valores, en la práctica y en la organización educativa.

\section{REFERENCIAS}

Assessment and Teaching of 21st Century Skills (ATC21s). (2011a). A partnership to drive change and success in education [Una alianza para guiar del cambio y éxito en educación]. Recuperado de http://www.cisco.com/web/ strategy/docs/education/atc21s.pdf

ATC21s (2011b). Module 1: Using a developmental model to assess student learning. Developmental learning framewoks [Uso de un modelo en desarrollo sobre la evaluación del aprendizaje del estudiante]. Recuperado de http://www.atc21s.org/uploads/3/7/0/0/37007163/pd_module_1_for_web_2014.pdf

Anderson, L., Krathwohl, D. y Bloom, B. (2001). A Taxonomy For Learning, Teaching, and Assessing: A Revision of Bloom's Educational Objectives [Una taxonomía para aprender, enseñar y evaluar: Revisión de los objetivos educativos de Bloom]. Pearson Education.

Barker, Ch. (1996). Generalization Skills of 3 and 4 years olds: Assessment and Instruction Using a Vygotskian Framework [Habilidades de generalización de 3 a 4 años: Enseñanza y evaluación usando el marco teórico de Vygotsky] (Tesis de maestría]. Graduate Department of Education. University of Toronto.

Clarke, L. y Winch, C. (2006). A European skill framework? But what are skills? Anglo-Saxon versus German concepts [¿Un marco europeo sobre habilidades? ¿Qué son las habilidades? Conceptos angosajones vs. alemanes]. Journal 
of Education at Work, 19(3), 255-269. Recuperado de http://westminsterresearch.wmin.ac.uk/2715/1/ Clarke_\%26_Winch_2006_final.pdf

Connell, M., Scheridan, K. y Gardner, H. (2003). On Abilities and Domain [Sobre habilidades y dominio]. En R. Sternberg y E. Grigorenco (Eds.), The Psychology of Abilities, Competencies and Expertise [Psicología de las habilidades, competencias y experticia] (pp.93-125). Cambridge University Press. Doi: http:// dx.doi.org/10.1017/CBO9780511615801

Dreyfus, H. y Dreyfus, S. (1986). Mind over Machine: the power of human intuition and expertise in the era of the computer [La mente sobre la máquina: El poder de la intuición humana y la expertise en la era de la computadora]. New York: The Free Press.

Dreyfus, S. (2004). The five-stage model of adult skill acquisition [El modelo de cinco estados de adquisición de una habilidad en adultos]. Bulletin of Science Technology \& Society, 24(3), 177-181. doi: $10.1177 / 0270467604264992$

Dussel, I. (2004). La escuela y la diversidad: Un debate necesario. Revista Todavía. Recuperado de http:// www.revistatodavia.com.ar/todavia21/8.dusselnota.html

Griffin, P. y Care, E. (2014). Developing learner collaborative problem solving skills [Desarrollando habilidades de resolución de problemas en estudiantes]. Recuperado de https://sodas.ugdome.lt/bylos/GENERAL/8af7dd98d82c-4d81-90ed-7f912c0dfcf0.docx

Heritage, M (2015). Learning Progressions: Supporting Instruction and Formative Assessment [Progreso de aprendizaje: Soporte para la instrucción y la evaluación formativa]. Washington: Council of Chief State School Officers.

Jornet, J. y González, J. (2009). Evaluación criterial: Determinación de estándares de interpretación (EE) para pruebas de rendimiento. Estudios sobre Educación, 16. 103-123. Recuperado de http://www.uv.es/gem/gemhistorico/publicaciones/ Evaluacion_criterial_Determinacion_de_estandares_de_interpretacion_EE_para_pruebas_de_rendimiento_educativo.pdf

Lozano, A. y Herrera, J. (2013). Diseño de programas educativos basados en competencias. México: Editorial Digital ITESM.

Mangrulkar, L., Vince Whiatman, C. y Posner, C. (2001). Life skill approach to Child and Adolescent Healthy Human Development [Enfoque de habilidades para la vida para el desarrollo humano de la salud de la niñez y adolescencia]. Washington, DC: Pan American Health Organization. Recuperado de http://hhd.org/resources/ research/life-skills-approach-child-and-adolescent-healthy-human-development

Ministerio de Educación Pública (MEP). (2008). Programas de estudio de Orientación. III Ciclo. San José: Autor.

Ministerio de Educación Pública MEP. (2009). Programa de estudio de Orientación. Educación Diversificada. San José: Autor.

Ministerio de Educación Pública MEP. (2015). Educar para una Nueva Ciudadanía: Transformación curricular. San José: Autor.

MECD (2013). Las competencias básicas: Una visión internacional desde el informe OECD "Sinergias para aprender mejor: Perspectiva internacional sobre evaluación". Recuperado de http://blog.educalab.es/inee/2013/06/19/las-competencias-basicas-una-vision-internacionaldesde-el-informe-ocde-sinergias-para-aprender-mejor-perspectiva-internacional-sobre-evaluacion/

Organización para la Cooperación y el Desarrollo Económicos OCDE. (2013). Draft Collaborative Problem Solving Framework [Borrador: Marco conceptual de la resolución colaborativa de problema]. Madrid: MECD. Recuperado de http://www.oecd.org/pisa/pisaproducts/Draft\%20PISA\%202015\%20Collaborative \%20Problem\%20Solving\%20Framework\%20.pdf

Organización para la Cooperación y el Desarrollo Económicos (OCDE). (2005). La definición y selección de competencias clave. Resumen Ejecutivo. Recuperado de http://deseco.ch/bfs/deseco/en/ index/03/02.parsys.78532.downloadList.94248.DownloadFile.tmp/2005.dscexecutivesummary.sp.pdf

Organización para la Cooperación y el Desarrollo Económicos OCDE. (2010). Habilidades y competencias del siglo XXI para los aprendices del nuevo milenio en los países de la OCDE. Instituto de 
Tecnologías Educativas. Recuperado de http://www.itm.edu.co/autoevaluacioninstitucional/ITMCifras/ Otros/Habilidades_y_competencias_siglo21_OCDE.pdf

Ornstein, A. y Hunkins, F. (2009). Curriculum: foundations, principles and issues [Curriculum: Fundamentos, principios y temas] (5ta ed.). Estados Unidos: Pearson.

Programa Estado de la Nación. (2013). Cuarto Informe Estado de la Educación. San José: Editorama.

Rigby, M. y Sanchis, E. (2006). The concept of skills and its social construction [El concepto de habilidad y su construcción social]. European Journal of vocational training, 37(1), 22-33.

Sturgis, C. (2015). Implementing Compentency Education in K-12 Systems: Insights from Local Leaders [Implementando una educación por competencias en el sistema educativo k-12: ideas de los líderes locales]. International Association for K-12 Online Learning. Recuperado de http://www.competencyworks.org/ resources/new-release-implementing-competency-education-in-k-12-systems-insights-from-local-leaders-2/

Sahlberg, P. (2006). Models of curriculum development: international trends and the way forward [Modelos de desarrollo curricular: Tendencias internacionales y camino a seguir]. Trabajo presentado en la Conferencia Internacional sobre reformas curriculares e implementación en el siglo XXI, Instanbul, Turquía.

Trilling, B. y Fadel, C. (2009). 21st century skills: learning for life in our times [Habilidades del siglo XXI: Aprender para la vida en nuestros tiempos]. San Francisco: Jossey-Bass.

UNICEF. (2005). Life Skills-Based Education in South Asia. Recuperado de http://www.unicef.org/rosa/Life_skillsbased_education_in_south_asia.pdf

UNESCO. (2004). Inter-Agency Working Group on Life Skills in EFA [Grupo Inter-Agencias sobre habilidades para la vida en EFA]. Paris: UNESCO.

World Health Organization (WHO). (1997). Life skills education for children and adolescents in school [Educación de habilidades para la vida en escuelas para niños y adolescentes]. Division of Mental Health and Prevention of Substance Abuse. Geneva: WHO.

WHO. (1999). Conclusions form a United Nations Inter-Agency Meeting. [Conclusiones del encuentro InterAgencias de las Naciones Unidas]. Department of Mental Healt. Geneva: WHO.

\section{BY-NC-ND}

